\title{
ABSTRACT \\ OBSERVATION OF EIT IN RUBIDIUM VAPOR USING THE HANLE EFFECT
}

\author{
By Yuhong (Iris) Zhang
}

In this thesis, I experimentally investigated Electromagnetically Induced Transparency (EIT) using the Hanle effect in Rubidium (Rb) atomic vapor. The Hanle effect results in a sharp coherent feature in the absorption of the incident beam at zero magnetic field. We have observed this Hanle peak with good signal-to-noise ratio in the $\operatorname{Rb} 87 F=1 \rightarrow F^{\prime}=1$ transition. Hanle features in this particular transition of $\mathrm{Rb}$ have never been previously observed. Our goal is to use the Hanle effect as a sensitive measure of radiation trapping. 


\title{
OBSERVATION OF EIT IN RUBIDIUM VAPOR USING THE HANLE EFFECT
}

\author{
A Thesis \\ Submitted to the \\ Faculty of Miami University \\ in partial fulfillment of \\ the requirements for the degree of \\ Master of Science \\ Department of Physics \\ by \\ Yuhong (Iris) Zhang \\ Miami University \\ Oxford, Ohio \\ 2007
}

Reader

Perry R. Rice

Reader

S. Burçin Bayram

Advisor

Samir Bali 


\section{Table of Contents}

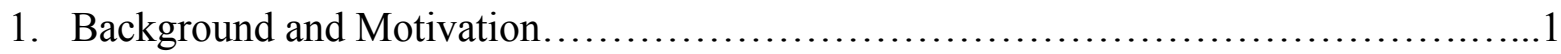

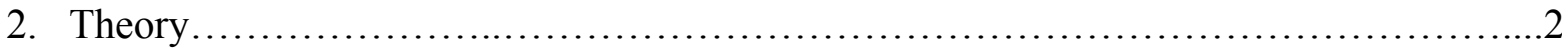

2.1 Lorentz Model.............................................................

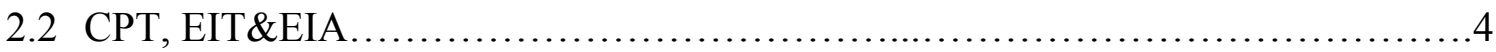

3. Experiment Setup and Operation............................................. 10

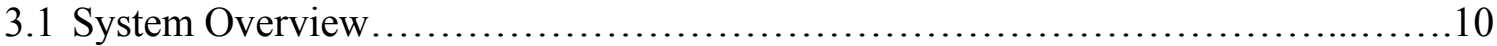

3.2 External Cavity Diode Laser.............................................12

3.2.1 Design........................................................... 12

3.2.2 Components and Alignment........................................ 13

3.2.3 Operation an maintenance......................................... 16

3.3 Saturated Absorption System........................................... 17

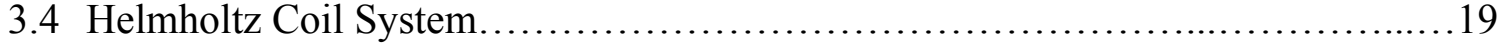

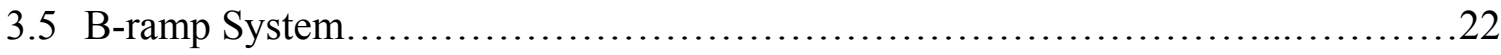

3.6 Operation of experiment.............................................. 24

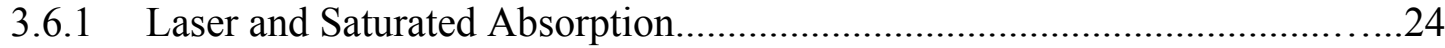

3.6.2 Hanle experiment.............................................27

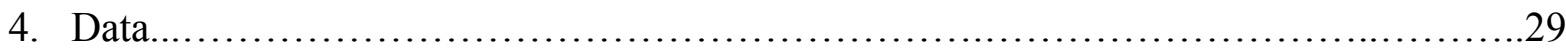

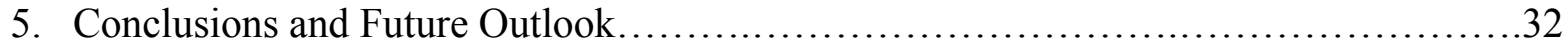

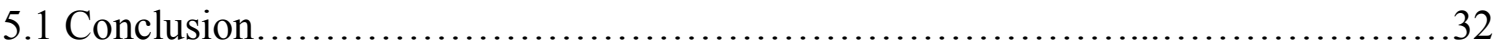

5.2 Future Outlook ............................................................

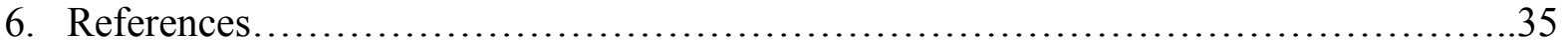




\section{Acknowledgements}

I would like to thank my advisor, Dr. Samir Bali, for all the training and support. He is my mentor in both research and personality. He played the most important role in my graduate career at Miami University. He made me an experimental physicist with critical ideas all the time.

I would also like to thank the members of my thesis committee, Dr. Perry Rice and Dr. Burcin Bayram. Thanks for their advice, help, and being the readers for this thesis.

And last, but certainly not least, I would like to thank my patients and fiancé Leon. Thank you for the understanding and the love you gave me. 
For my parents and Leon 


\section{Chapter 1 \\ Background \& Motivation}

EIT had a profound impact upon optical science [1]. By modifying the light and the media, we can turn a transparent medium into an opaque one. EIT can also be used in lasing without inversion [2]. When we incident coherent laser bean on an atomic medium and set it on resonance, the atoms will be pumped to new eigenstates due to light-atom interaction. If quantum interference happens between the excitation pathways, there will be no absorption from the coherent laser beam. This was termed electromagnetically induced transparency (EIT) by Harris and co-workers (Harris et al., 1990). EIT has been studied extensively for more than 25 years. The work in the field can be found, for example, Arimondo (1996), Harris (1997) or Scully (1992), and in the articles of Lukin, Hemmer, and Scully (2000); Matsko, Kocharovskaya, et al. (2001); Vitanov et al. (2001).

The phenomenon of coherent population trapping (CPT) was observed by Alzetta et al. (1976). CPT is closely related to EIT. When EIT happens the atoms are pumped to a new eigenstate, which leads to CPT. A review of CPT was published by Arimondo in 1996 [3].

The opposite effect of EIT, electromagnetically induced Absorption (EIA) was found by A. Lezama, S. Barreiro, and A. M. Akulshin in 1999. EIA shows an enhancement of the absorption resulting from atomic coherence induced by optical radiation. The phenomenon of EIA is in many aspects complementary to EIT. [4, 5]

Traditionally EIT is obtained by using two lasers with different frequencies tuned to energy levels suitable for EIT. But, reference $[6,7]$ showed that EIT can also be obtained by using a single frequency laser been tuned to different Zeeman sublevels by application of external B, which is Hanle configuration. The Hanle effect [8] enables a very simple geometry for EIT experiments. Our goal is to obtain EIT\&EIA in a simple Hanle configuration with good SNR. In this thesis, we present our experiment progress toward achieving this goal. 


\section{Chapter 2 \\ Theory}

In this chapter, we present the theory for coherent population trapping (CPT), electromagnetically induced transparency (EIT) \& electromagnetically induced absorption (EIA) in the context of the Hanle configuration. This is because our experiment is done using the Hanle configuration.

The Hanle effect refers to the use of a magnetic field to produce CPT between Zeeman atomic sublevels $[3,9]$. CPT arises from the optical pumping of Zeeman sublevels of the ground state to an excited state by a single linearly polarized incident laser beam. The new eigenstate, which the atoms are pumped into, is known as the dark state [10]. It results in a dip in the absorption signal at zero magnetic field, which leads to Electromagnetically Induced Transparency (EIT).

In this chapter, we first describe the Lorentz model in Section 2.1, which forms the basis for treating the atom as a dipole. Next, in section 2.2, I will describe CPT, EIT\&EIA. 


\subsection{Lorentz Model}
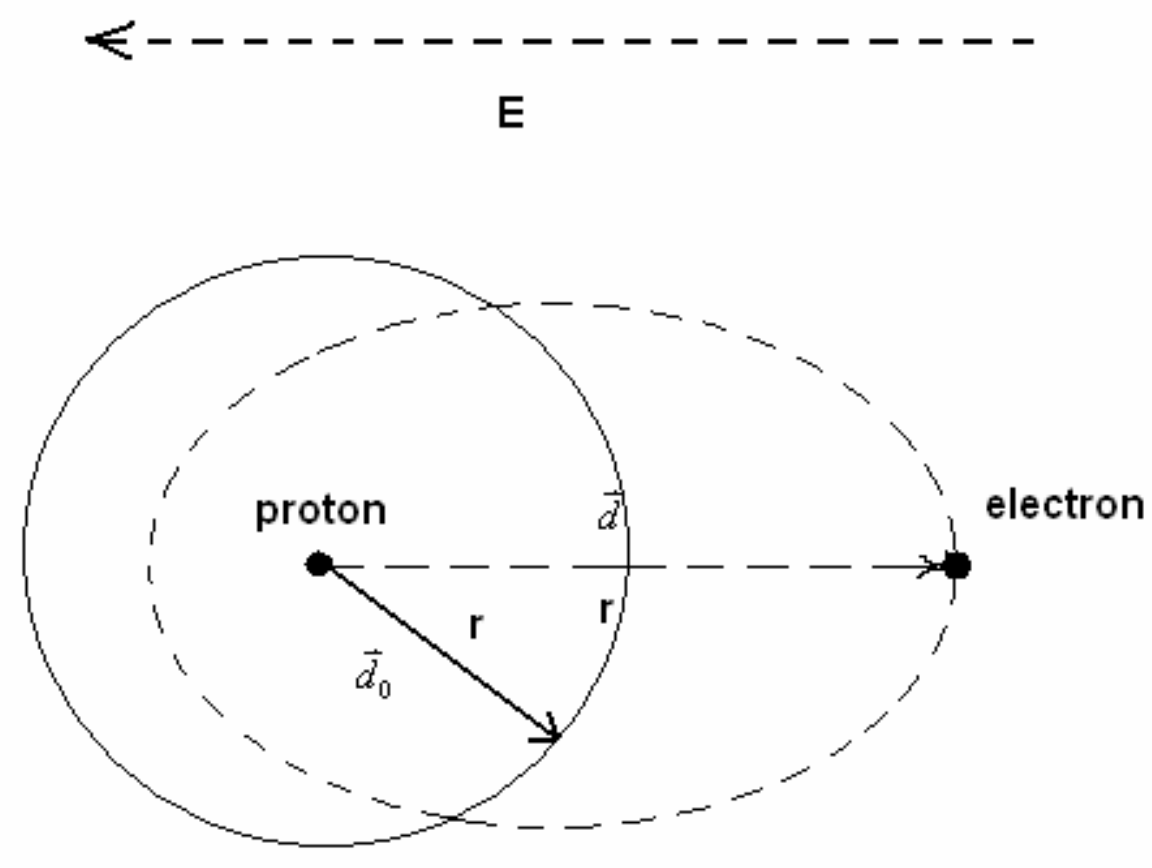

Figure 2.1: Lorentz Model of Light. Solid lines denote atom in the absence of E field; dashed lines denote atom in the E field.

Figure 2.1 shows us the Lorentz Model of Light - Atom interaction. $\vec{d}$ is the dipole moment, $\vec{d}=e \vec{r}$. Average dipole moment in the absence of light or field is $\left\langle\vec{d}_{0}\right\rangle=e\langle\vec{r}\rangle=0$. After shining field $\vec{E},\langle\vec{d}\rangle \neq 0$.

In the present of the field, the Hamiltonian $(H)$ is $H=H_{A}+H_{F}+H^{\prime}$, where $H^{\prime}=-\vec{d} \cdot \vec{E}$ is the atom-field interaction Hamiltonian; $H_{A}$ is the time-independent atomic Hamiltonian in the absence of light; $H_{F}$ is the free field Hamiltonian, which shows the field. 


\subsection{CPT, EIT\&EIA}

When we place an atomic system in a magnetic field, the hyperfine energy levels are split into multiple sublevels, which energies are changed proportionally to the magnetic field strength. These energy shifts are known as the Zeeman Effect. The earth's magnetic field, for instance, is enough to produce a measurable Zeeman shift.

Consider linearly polarized light incident on a $J=1 \rightarrow J^{\prime}=0$ atom, with Zeeman sublevels m, as shown in Fig. 2.2.1 $J$ is the total electron angular momentum. $\vec{J}=\vec{L}+\vec{S}$, $\mathrm{L}$ is orbital electron angular momentum. $\mathrm{S}$ is electron spin. $\vec{F}=\vec{J}+\vec{I}$ is the total angular momentum of the atom. $I$ is the nuclear spin, assumed zero in the following discussion for simplicity.
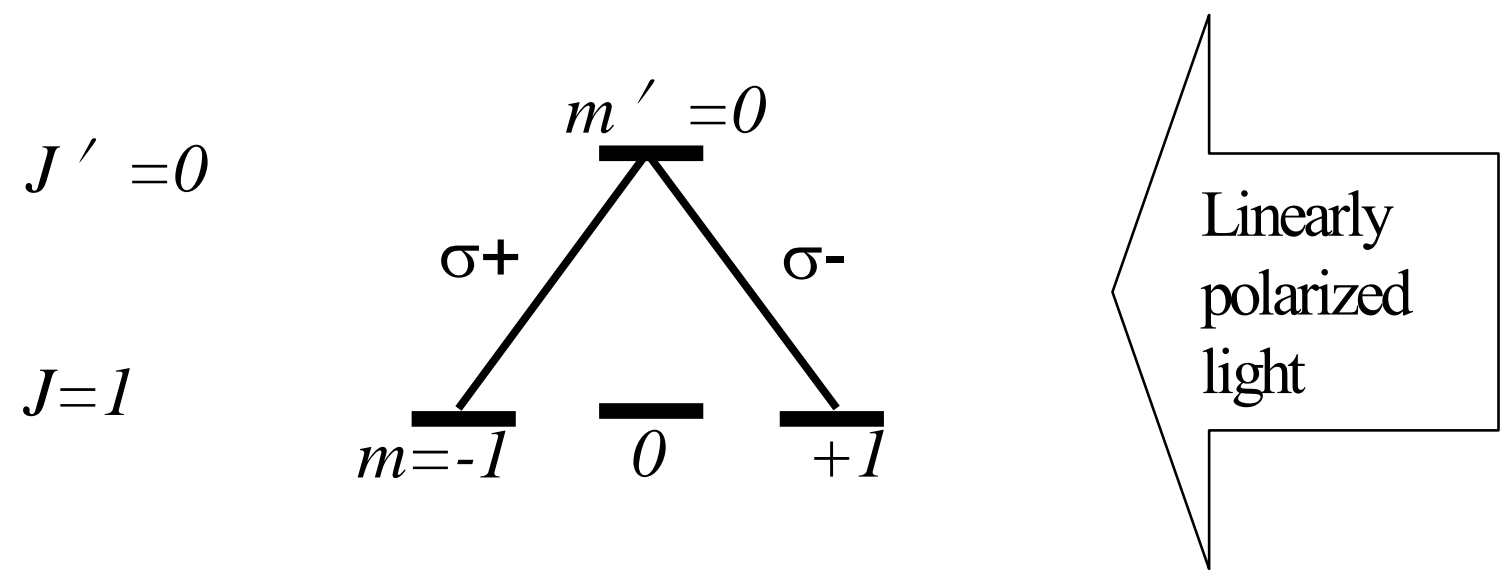

Figure 2.2.1: The Hanle configuration for observing EIT

If we apply B field in $\mathrm{z}$ direction, the axis of quantization will be in $\mathrm{z}$ direction. We apply linearly polarized light traveling in $\mathrm{z}$ direction, so that the beam is polarized in $\mathrm{x}$ or $\mathrm{y}$ direction. The quantization axis of the atom is different from the linear polarization of the laser beam so the atom won't see the linearly polarized light. We can consider the linear polarized light as the superposition of $\sigma^{+}$and $\sigma^{-}$light. Based on the dipole selection rules, $\Delta m=+1$ is for $\sigma^{+}$light and $\Delta m=-1$ is for $\sigma^{-}$light in absorption as shown in 
Figure 2.2.1. If our light is linearly polarized in $\mathrm{x} / \mathrm{y}$ direction, we have $\hat{x}=\frac{1}{\sqrt{2}}\left(\hat{\varepsilon}_{+}+\hat{\varepsilon}_{-}\right) / \hat{y}=\frac{i}{\sqrt{2}}\left(\hat{\varepsilon}_{+}+\hat{\varepsilon}_{-}\right)$.

$\hat{x}$ and $\hat{y}$ are Cartesian vector basis, $\hat{\varepsilon}_{+}$and $\hat{\varepsilon}_{-}$are cylindrical vector basis, which are $\hat{\varepsilon}_{+}=\frac{1}{\sqrt{2}}(\hat{x}+i \hat{y})$ for $\sigma^{+}$light and $\quad \hat{\varepsilon}_{-}=\frac{1}{\sqrt{2}}(\hat{x}-i \hat{y})$ for $\sigma^{-}$light.

Use $\hat{x}=\frac{1}{\sqrt{2}}\left(\hat{\varepsilon}_{+}+\hat{\varepsilon}_{-}\right)$and $\hat{y}=\frac{i}{\sqrt{2}}\left(\hat{\varepsilon}_{+}+\hat{\varepsilon}_{-}\right)$, we may write the dipole operator $\vec{d}$ as $\vec{d}=d_{x} \hat{x}+d_{y} \hat{y}+d_{z} \hat{z}=d_{z} \hat{z}+d_{+} \hat{\varepsilon}_{+}+d_{-} \hat{\varepsilon}_{-} \quad, \quad$ where $\quad d_{+}=-\frac{1}{\sqrt{2}}\left(d_{x}-i d_{y}\right) \quad$ and $d_{-}=\frac{1}{\sqrt{2}}\left(d_{x}+i d_{y}\right)$

Similarly $E_{+}=-\frac{1}{\sqrt{2}}\left(E_{x}-i E_{y}\right)$ and $E_{-}=\frac{1}{\sqrt{2}}\left(E_{x}+i E_{y}\right)$. Then we have

$$
\begin{aligned}
& H^{\prime}=-\vec{d} \cdot \vec{E}=-\left\{\left(d_{x} \hat{x}+d_{y} \hat{y}+d_{z} \hat{z}\right) \cdot\left(E_{x} \hat{x}+E_{y} \hat{y}+E_{z} \hat{z}\right)\right\} \\
& =-\left(d_{x} E_{x}+d_{y} E_{y}+d_{z} E_{z}\right)=-d_{z} E_{z}+d_{-} E_{+}+d_{+} E_{-}
\end{aligned}
$$

Let initial state $|i\rangle=C_{-1}|J=1, m=-1\rangle+C_{0}|J=1, m=0\rangle+C_{1}|J=1, m=1\rangle$

$$
=C_{-1}|-1\rangle+C_{0}|0\rangle+C_{1}|1\rangle
$$

And final state $|f\rangle=\left|J^{\prime}=0, m^{\prime}=0\right\rangle=|0\rangle$,

so $\left\langle f\left|H^{\prime}\right| i\right\rangle=\left\langle 0\left|\left(-d_{z} E_{z}+d_{-} E_{+}+d_{+} E_{-}\right)\right|\left(C_{-1}|-1\rangle+C_{0}|0\rangle+C_{1}|1\rangle\right)\right\rangle$

If light is polarized in x direction, we have $\vec{E}=E_{x} \hat{x}=\hat{x} E_{0} \cos \omega t ; \vec{E}_{y}=\overrightarrow{0} ; \vec{E}_{z}=\overrightarrow{0}$, $H^{\prime}=-\frac{1}{\sqrt{2}} d_{-} \vec{E}_{x}+\frac{1}{\sqrt{2}} d_{+} \vec{E}_{x}=\frac{1}{\sqrt{2}} E_{0} \cos \omega t\left(d_{+}-d_{-}\right)$ 
Then we have Equation $(1)=\frac{E_{0}}{\sqrt{2}} \cos \omega t\left[\left\langle 0\left|\left(d_{+}-d_{-}\right)\right|\left(C_{-1}|-1\rangle+C_{0}|0\rangle+C_{1}|1\rangle\right)\right\rangle\right]$

$\Rightarrow\left\langle f\left|H^{\prime}\right| i\right\rangle \propto C_{-1}\left\langle 0\left|d_{+}-d_{-}\right|-1\right\rangle+C_{1}\left\langle 0\left|d_{+}-d_{-}\right| 1\right\rangle+C_{0}\left\langle 0\left|d_{+}-d_{-}\right| 0\right\rangle$

For x polarized light, we have $\left\langle f\left|H^{\prime}\right| i\right\rangle \propto C_{1}\left\langle 0\left|d_{+}\right| 1\right\rangle-C_{-1}\left\langle 0\left|d_{-}\right|-1\right\rangle$

For y polarized light, $\vec{E}=E_{y} \hat{y}=E_{0} \cos \omega t \hat{y}$, going through a similar process,

$$
H^{\prime}=\frac{i}{\sqrt{2}} E_{0} \cos \omega t\left(d_{+}+d_{-}\right) \text {and }\left\langle f\left|H^{\prime}\right| i\right\rangle \propto C_{1}\left\langle 0\left|d_{+}\right| 1\right\rangle+C_{-1}\left\langle 0\left|d_{-}\right|-1\right\rangle
$$

We know that $\left\langle 0\left|d_{+}\right| 1\right\rangle=\left\langle J^{\prime}=0, m^{\prime}=0\left|d_{+}\right| J=1, m=1\right\rangle$

$$
\left\langle 0\left|d_{-}\right|-1\right\rangle=\left\langle J^{\prime}=0, m^{\prime}=0\left|d_{-}\right| J=1, m=-1\right\rangle
$$

Using Wigner-Eckart Theorem [11] and standard 3-J symbol terminology [12]

$$
\left\langle r^{\prime}, J^{\prime}, m^{\prime}\left|T_{q}^{k}\right| r, J, m\right\rangle=\left\langle r^{\prime}, J^{\prime}\|d\| r, J\right\rangle(-1)^{J^{\prime}-m^{\prime}}=\left(\begin{array}{c}
J^{\prime} k J \\
m^{\prime} q m
\end{array}\right)
$$

$\mathrm{T}$ is the dipole tensor; $\mathrm{k}$ is the rank of tensor ( $\mathrm{k}=1$ for dipole); $\mathrm{q}$ is the polarization $\left(q= \pm 1\right.$ for $\sigma^{ \pm}$light $)$

For the $\sigma^{+}$light, the $d_{-}$term is $\left(\begin{array}{ccc}0 & 1 & 1 \\ 0 & 1 & -1\end{array}\right)$

For the $\sigma^{-}$light, the $d_{+}$term is $\left(\begin{array}{ccc}0 & 1 & 1 \\ 0 & -1 & 1\end{array}\right)$

In general, this reduces to,

$$
\left(\begin{array}{llc}
a & b & a+b \\
c & d & -c-d
\end{array}\right)=(-1)^{a-b+c+d}\left[\frac{(2 a) !(2 b) !(a+b+c+d) !(a+b-c-d) !}{(2 a+2 b+1) !(a+c) !(a-c) !(b+d) !(b-d) !}\right]^{\frac{1}{2}}
$$

And the Wigner 3-J symbols have the symmetries:

$$
\left(\begin{array}{ccc}
j_{1} & j_{2} & J \\
m_{1} & m_{2} & M
\end{array}\right)=\left(\begin{array}{ccc}
j_{2} & J & j_{1} \\
m_{2} & M & m_{1}
\end{array}\right)=\left(\begin{array}{ccc}
J & j_{1} & j_{2} \\
M & m_{1} & m_{2}
\end{array}\right)=\left(\begin{array}{ccc}
j_{1} & j_{2} & j_{1}+j_{2} \\
m_{1} & m_{2} & -M
\end{array}\right)
$$


Then Equation $(2)=(-1)^{j_{1}-j_{2}+M}\left[\frac{\left(2 j_{1}\right) !\left(2 j_{2}\right) !\left(j_{1}+j_{2}+M\right) !\left(j_{1}+j_{2}-M\right) !}{\left(2 j_{1}+2 j_{2}+1\right) !\left(j_{1}+m_{1}\right) !\left(j_{1}-m_{1}\right) !\left(j_{2}+m_{2}\right) !\left(j_{2}-m_{2}\right) !}\right]^{\frac{1}{2}}$

For both $\sigma^{+}$and $\sigma^{-}$light, the 3-J symbol is:

$\left(\begin{array}{ccc}0 & 1 & 1 \\ 0 & 1 & -1\end{array}\right)=(-1)^{1-1}\left[\frac{(2 \times 0) !(2 \times 1) !(0+1+0+1) !(0+1-0-1) !}{(2 \times 0+2+1) !(0) !(0) !(1+1) !(1-1) !}\right]^{\frac{1}{2}}=\left(\frac{1}{3}\right)^{\frac{1}{2}}=\frac{1}{\sqrt{3}}$

For x polarized light $\left\langle f\left|H^{\prime}\right| i\right\rangle=\frac{E_{0}}{\sqrt{2}} \cos \omega t\left\{C_{1}\left\langle 0\left|d_{+}\right| 1\right\rangle-C_{-1}\left\langle 0\left|d_{-}\right|-1\right\rangle\right\}$

and for $y$ polarized light $\left\langle f\left|H^{\prime}\right| i\right\rangle=\frac{E_{0}}{\sqrt{2}} \cos \omega t\left\{C_{1}\left\langle 0\left|d_{+}\right| 1\right\rangle+C_{-1}\left\langle 0\left|d_{-}\right|-1\right\rangle\right\}$, where $d_{-}=b^{+}$, the atomic raising operator and $d_{+}=b^{-}$the atomic lowering operator. The action of these atomic lowing and raising operators is:

$b^{+}|0\rangle=|1\rangle ; b^{+}|1\rangle=0 ; b^{+}|-1\rangle=|0\rangle$

$b^{-}|0\rangle=|-1\rangle ; b^{-}|1\rangle=|0\rangle ; b^{-}|-1\rangle=0$

If $C_{1}=C_{-1}$, then $\left\langle f\left|H^{\prime}\right| i\right\rangle_{x \text { pol }}=\frac{E_{0}}{\sqrt{2}} \cos \omega t\left\{C_{1}\left\langle 0\left|b^{-}\right| 1\right\rangle-C_{1}\left\langle 0\left|b^{+}\right|-1\right\rangle\right\}$

$$
\begin{aligned}
& =\frac{E_{0}}{\sqrt{2}} \cos \omega t\left\{C_{1}\langle 0 \mid 0\rangle-C_{1}\langle 0 \mid 0\rangle\right\} \\
& =0
\end{aligned}
$$

$\left\langle f\left|H^{\prime}\right| i\right\rangle_{x p o l}=0$ results in dark state/non absorbing state. The fact that all atoms can be accumulated in the dark state is called coherent population trapping (CPT).

On the other hand $\left\langle f\left|H^{\prime}\right| i\right\rangle_{y \text { pol }}=\frac{E_{0}}{\sqrt{2}} \cos \omega t\left\{C_{1}\left\langle 0\left|b^{-}\right| 1\right\rangle+C_{1}\left\langle 0\left|b^{+}\right|-1\right\rangle\right\}$

$$
\begin{aligned}
& =\frac{E_{0}}{\sqrt{2}} \cos \omega t\left\{C_{1}\langle 0 \mid 0\rangle+C_{1}\langle 0 \mid 0\rangle\right\} \\
& =\frac{E_{0}}{\sqrt{2}} \cos \omega t\left\{2 C_{1}\langle 0 \mid 0\rangle\right\}
\end{aligned}
$$

$\left\langle f\left|H^{\prime}\right| i\right\rangle_{y \text { pol }}=\max$. results in bright state. 
Similarly, if $C_{1}=-C_{-1}$, then $\left\langle f\left|H^{\prime}\right| i\right\rangle_{x \text { pol }}=\max \cdot\left\langle f\left|H^{\prime}\right| i\right\rangle_{y \text { pol }}=0$, which means that we have bright state for x polarized light, but dark state for y polarized light.

Based on the calculation above, when $B \neq 0$, atoms are pumped to excited states. Fluorescence from different Zeeman sublevels will produce light of different frequencies. When $\mathrm{B}=0$ or the Zeeman shift is at a minimum, Zeeman sublevels become degenerate $\left(C_{1}=-C_{-1}\right.$ or $C_{1}=C_{-1}$ ), the atom is pumped by $\sigma^{+}$light and $\sigma^{-}$lights into a new eigenstate (Dark state), which leads to Coherent Population Trapping (CPT) in the dark state and hence no absorption! As $|\mathrm{B}|$ increases, the dark state is destroyed. If we record the Absorption versus $\mathrm{B}$, we find a dip in the fluorescence intensity at $\mathrm{B}=0$, which shows EIT (Fig. 2.2.2) [6, 7]. The method described above is based on the Hanle Effect [9]. Basically the Hanle effect allows us to use an appropriately aligned B-field and a singlelinearly polarized laser beam to achieve EIT in a particularly simple manner.

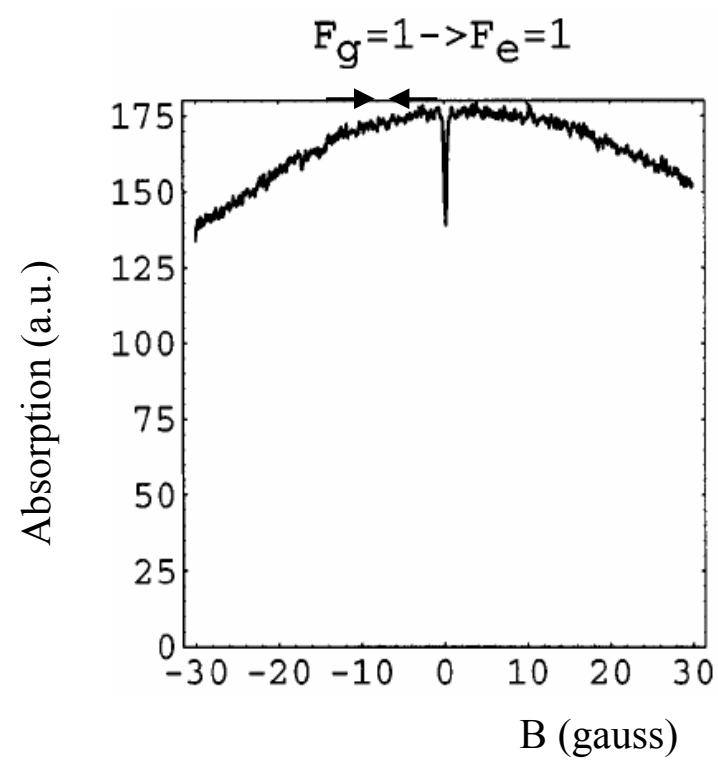

Figure 2.2.2: Reproduced from Ref. [6] -Typical Data for the $F_{g}=1 \rightarrow F_{e}=1$ transition in sodium. EIT occurs at $\mathrm{B}=0$ in Absorption. 
Similarly, if we use $J=0 \rightarrow J^{\prime}=1$ system, the dark state occurs in the excited state, and then we have electromagnetically induced absorption (EIA), see figure 2.2.3.

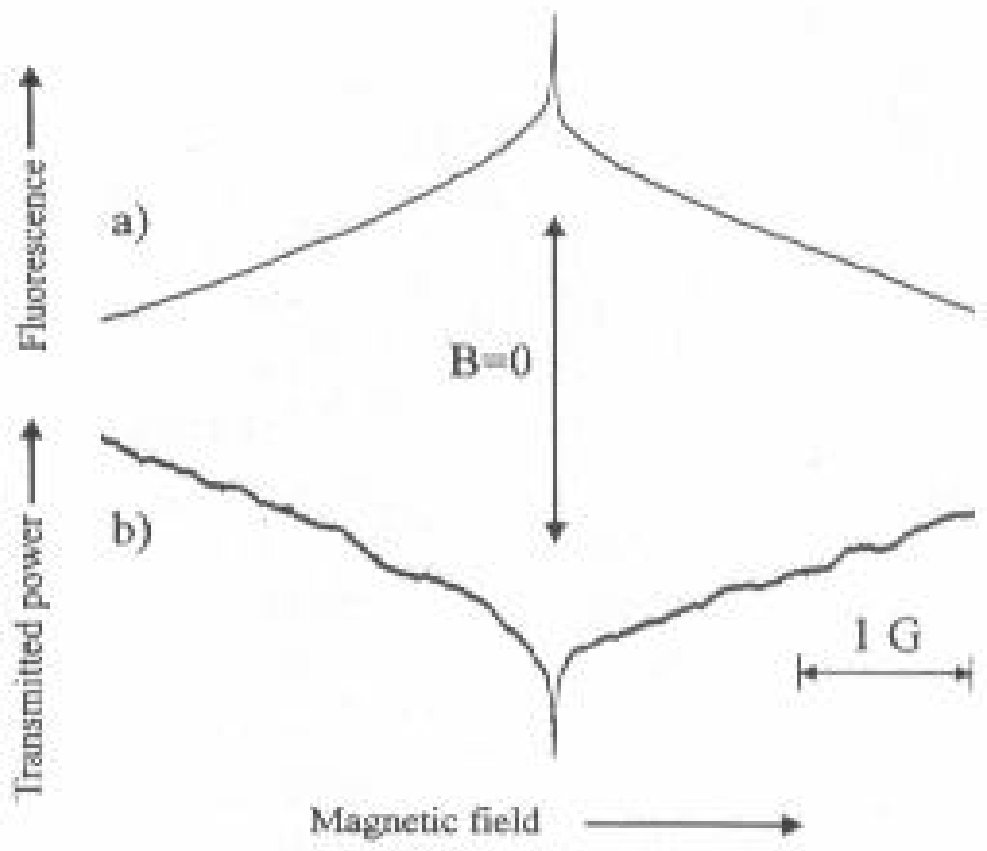

Figure 2.2.3: Results reproduced from reference [8]. The top curve represents fluorescence, while the bottom curve represents transmission. EIA occurs at $\mathrm{B}=0$ in fluorescence. 


\section{Chapter 3 \\ Experiment Setup and Operation}

This chapter shows the readers the way we built the Hanle configuration. Technical details are included.

\subsection{System Overview}

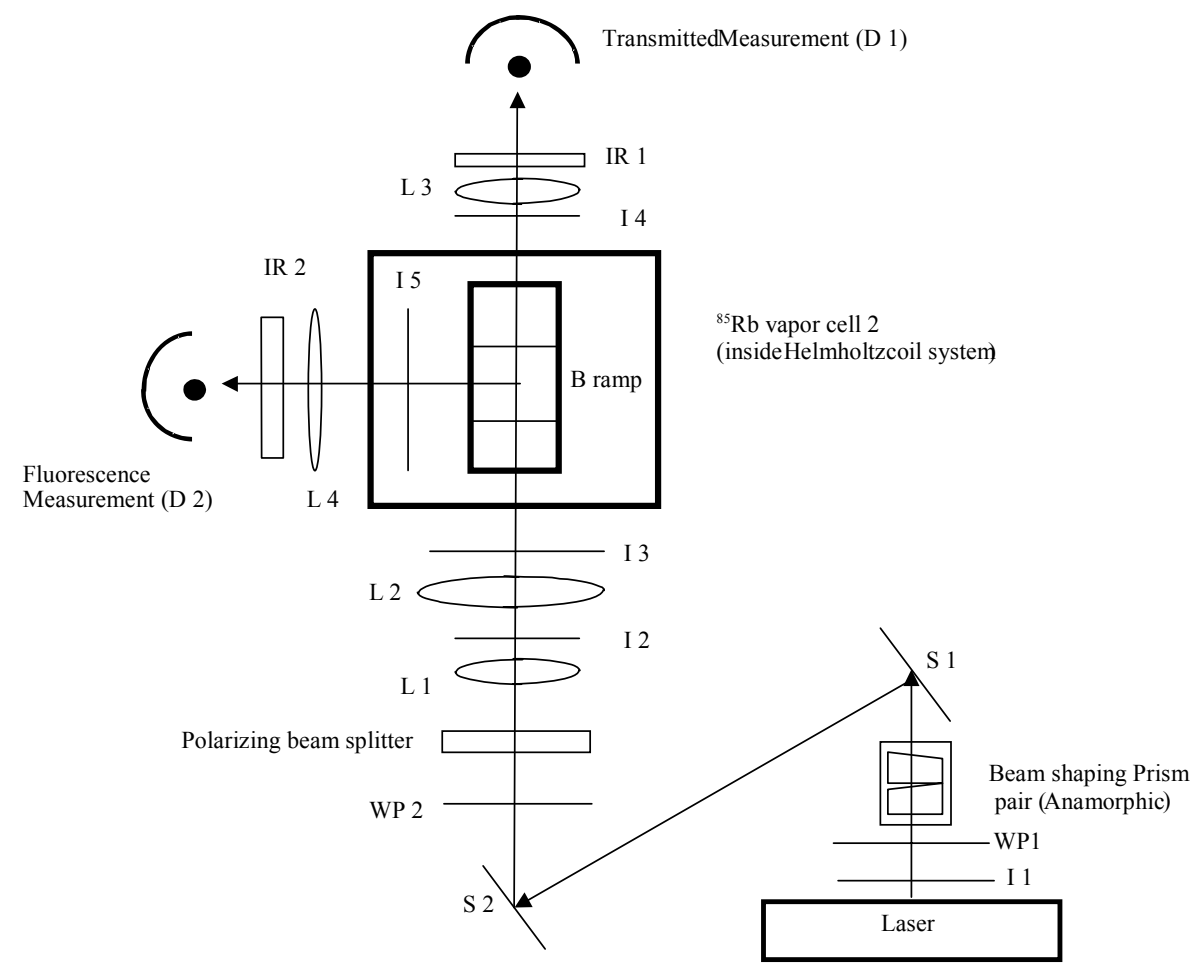

Figure 3.1 Experiment setup. I=Iris, WP=half-wave plate, $\mathrm{S}=$ steering mirror, $\mathrm{L}=$ lens, $\mathrm{IR}=$ infrared filter, $\mathrm{D}=$ detector, $\mathrm{f}=$ focus, $\mathrm{f}_{1}<10 \mathrm{~mm}, \mathrm{f}_{2}=160 \mathrm{~m}, \mathrm{f}_{3}=25 \mathrm{~mm}, \mathrm{f}_{4}=25 \mathrm{~mm}, \mathrm{l}=$ incident beam size, $1=20 \mathrm{~mm}$ 
Figure 3.1 shows the schematic Experimental Setup. We made a tunable frequency laser system using an external cavity diode laser. Then shine the laser into saturated absorption system and get the spectrum for $\mathrm{Rb}^{85}$ and $\mathrm{Rb}^{87}$. First, we incident the laser into beam shaping prism pairs to convert the elliptical beam shape into a round one. By adjusting the $\lambda / 2$ - plate (P1), we can minimize the intensity loss through the beam shaping [refer to Benjamin Agyare's thesis]. Then we use two steering mirrors to control the incident direction of the laser beam. The $\lambda / 2$ - plate (P2) and the Polarizing beam splitter make the incident intensity the sample adjustable. The Irises and the lenses expand the incident beam size from $2 \mathrm{~mm}$ to $20 \mathrm{~mm}$. The sample is a Rubidium vapor cell inside a 3-D Helmholtz coil system shielding from the earth magnetic field. We can sweep the amplitude of a B-field applied to the atoms in the vapor cell. We use two photodiodes to receive transmitted and fluorescence signals from the cell. To protect the whole system from outside disturbance, like unwanted light and air current, we built an aluminum frame with black cloth curtains on the top of the optical table. The frame can also hold lots of instruments used by the setup.

In the following subsections, we will describe the External Cavity Diode Laser, which is the light source of the experiment, the Saturated Absorption setup, which allows us to do frequency tuning of the light source, the Helmholtz coil system, B-ramp system, and the operation of the experiment. 


\subsection{External Cavity Diode Laser}

Our experiment needs a tunable frequency laser system to provide light source. Due to the high price of such kind of laser system with lower frequency tuning characteristic in the market, we decided to build one by ourselves. Diode lasers are quite cheap and are widely used in atomic physics [13]. The band width of our laser system is smaller than 1 MHz. In order to continuously tune the laser over larger range, we used standard optical feedback techniques [14]. The feedback is provided by a diffraction grating. By applying a ramp voltage to a PZT attached to the diffraction grating, we can continuously scan over 1.5 2GHz. In this section, we will describe the design and the components of the external cavity diode laser system.

\subsubsection{Design}

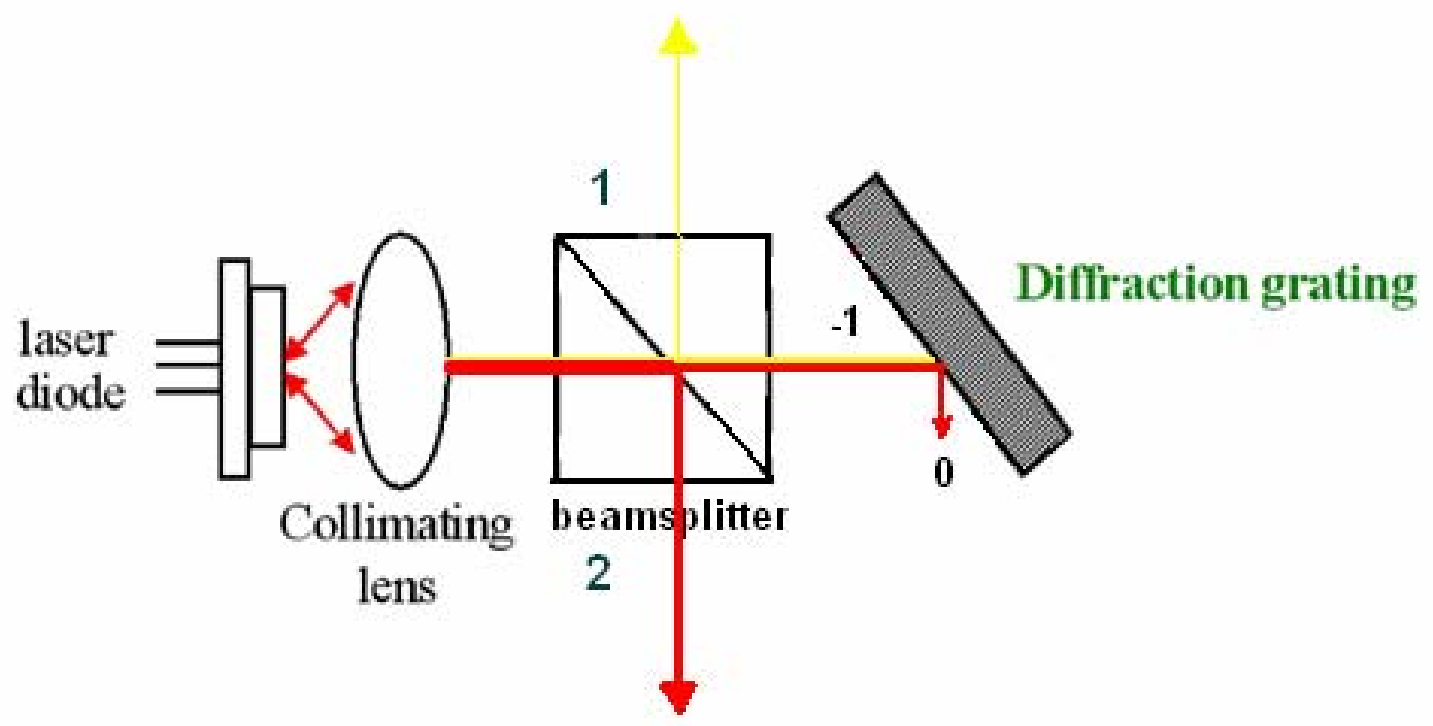

Figure 3.2.1: Basic design of the external cavity diode laser

Figure 3.2.1 shows the basic design of the laser system. We put the laser diode at the focus of a collimating lens so that the laser beam will focus on infinity at the other side of the lens. A non-polarizing beamsplitter is put after the collimating lens. It transmits half of the collimated light and reflects the other half. The transmitted collimated light hits a 
diffraction grating and come back to the beamsplitter. The beamsplitter again transmits half of the light, and reflects the other half. The diffraction grating is rotatable. It provides frequency selection feedback for the laser diode. We can adjust the angle of the grating and reflect the -1 order diffraction beam back to the laser through the beamsplitter. As a result of these, we can find reflected outputs from sides 1 and side 2. We will use the weaker output for Saturated Absorption setup, and use the stronger one for the experiment part.

\subsubsection{Components and Alignment}

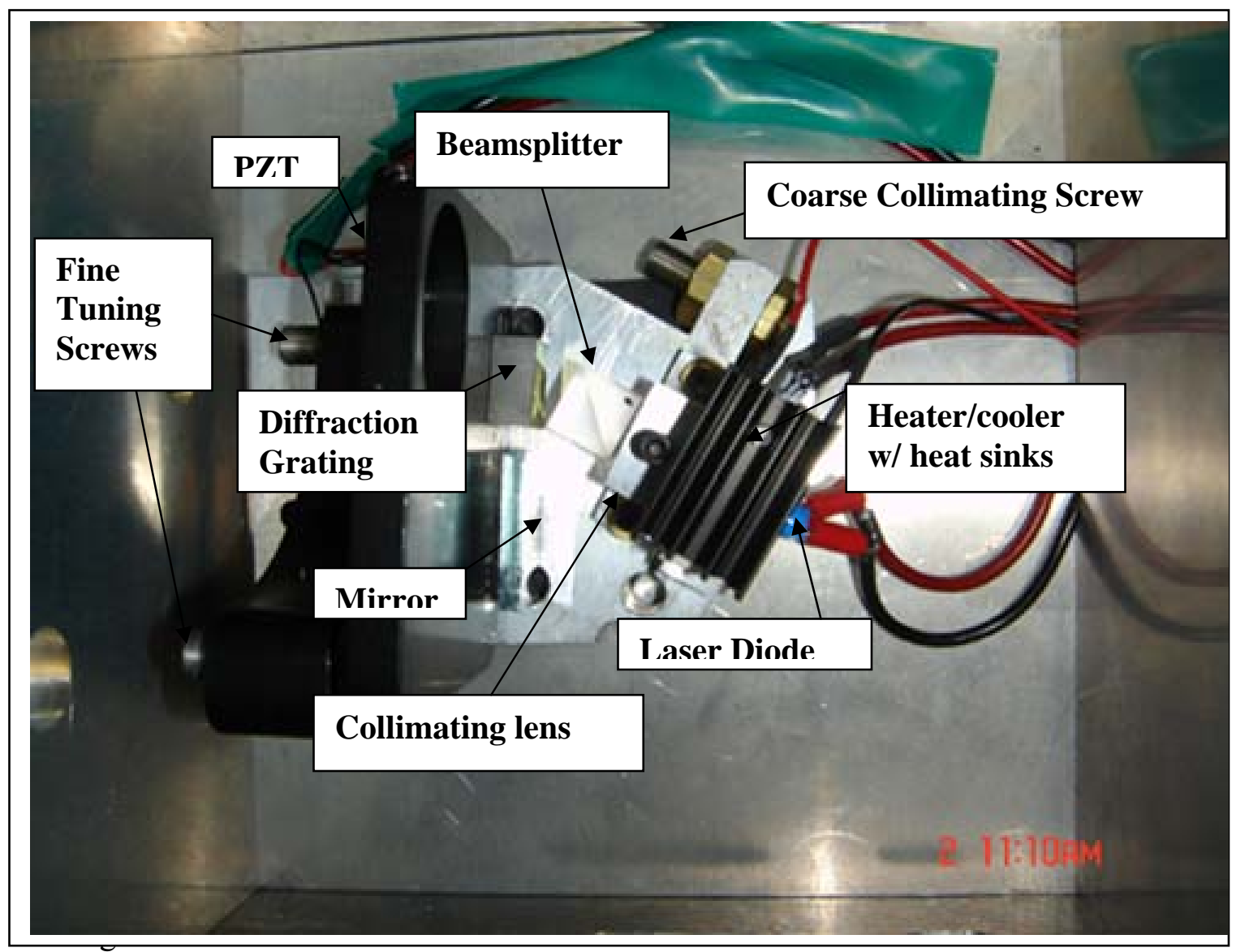

Figure 3.2.2: Actual setup of external cavity diode laser 
Figure 3.2.2 shows you the actual setup of laser system. We mount all the devices on a baseplate. We put sorbothane between baseplate the optical table to reduce vibration. And then we cover the devices with aluminum walls. The laser diode is mounted in laser housing. The laser housing is set on a Teflon spacer, then to the base plate. The Teflon spacer can thermally isolate the laser housing from the rest of the setup. Beside this, we use Nylon screws to prevent any heat conduction.

We are using diode laser from Sharp. The manufacturer part number GH0781JA2C, Its maximum optical power output is $120 \mathrm{~mW}$ at $140 \mathrm{~mA}$. The center wavelength is $784 \mathrm{~nm}$. The unit price is $\$ 24.76$. We orient the laser diode such that the longer axis of the elliptical beam is parallel to the table. Then the polarization direction is perpendicular to the table.

In order to temperature tune the laser to assist in frequency tuning, we drilled a hole closely enough to the diode laser on the laser housing, then place a $10 \mathrm{k} \Omega$ thermistor to sense the temperature change of the laser. The temperature signal will be sent to the temperature controller (Model: ILX Lightwave LC-3900 MODULAR Laser diode controller) and the corresponding calibrated current will be sent back to the heater/cooler to compensate the temperature change. Thus the whole temperature control system includes a thermistor, a temperature controller and heater/cooler. The laser diode is easily brought on resonance with $\mathrm{Rb}$ vapor typically at $26.2^{\circ} \mathrm{C}$ with a current of $78-81 \mathrm{~mA}$. After the diode laser, a collimating lens $(f=5 \mathrm{~mm}$, numerical aperture 0.5 , from Rodenstock/LINOS Photonics) is mounted between the diode laser and the grating. The lens is antireflective coated for $600-1020 \mathrm{~nm}$. The center of the lens should be at the same height as the center of the laser diode to ensure the beam is at constant height. The distance of the lens from the laser diode is controlled by a micrometer screw.

When we do the coarse collimation for the ECDL (external cavity diode laser), we place a white paper as far as possible away (at least a few meters) from the lens and look at the image of the beam on the paper, and then adjust the micrometer screw until we focus the beam on the paper, then we tune the lens a little bit closer to the diode laser so that the 
beam focus on infinity. Ideally the beam should have a constant size and shape over this distance of a few meters.

Next step, we glue a beamsplitter onto a $1 \mathrm{~cm}^{3}$ aluminum cube and place it between the lens and the grating. Then we solder two piezoelectric disks back to back and put them between the horizontal-tuning knob and the back of the front plate of the mount holding the diffraction grating. Between the metal mount of the grating and the piezoelectric disks, we need to stick plastic spacers to electrically isolate them form each other. Piezos can be found in buzzers. Plastic spacers can be cut from transparency sheets. This setup allows us to finely adjust the angle of the grating by applying a voltage ramp from a function generator. When the piezos flex, we can move the diffraction grating by very small amounts.

Next, we try to feed the first order from the diffraction grating back to the laser diode. We will see two spots out of side 1 or side 2. (We use the weak output side.) The spots are the beams reflected from the diffraction grating. One spot is after a single pass through the beamsplitter. The other spot is after double passes through the beamsplitter. When we slightly move the grating, the single pass does not move much while the double pass moves a lot. Then we move these two spots closer to each other by adjusting the angle of the grating mount until they jump to each other. At this time we know the light is returning directly back to the laser diode. If we continue tuning the angle of the grating mount, the two spots will overlap each other for a small range of adjustments. (After that they will separate again.) By this means, we are tuning the frequency of the laser diode with the help of external feed back. 


\subsubsection{Operation and maintenance}

We need to lower the threshold of the laser to get maximum feedback every day after we turn on the laser.

First, bring down the current of the laser until the laser is just at the threshold. Then bring down the current a little bit so the laser stops lasing.

Second, touch the vertically adjustment screw until the laser lases again. Then bring down the current so the laser stops lasing again.

Third, touch the horizontal adjustment screw until the laser lases again. Then bring down the current so the laser stops lasing again.

Forth, go back to touch the vertical and then horizontal adjustment screw using the same technique until the threshold can not be lowered anymore.

Fifth, place tapes over the adjustment holes to minimize any external influences, and open them only when need.

When the laser of this experiment was built one year ago, the threshold was $18 \mathrm{~mA}$ at $25.1^{\circ} \mathrm{C}$. After the winter came, the threshold changed to $19.3 \mathrm{~mA}$ at $26^{\circ} \mathrm{C}$. Now after we fixed the piezoes in the ECDL, the threshold is $22 \mathrm{~mA}$ at $25.8^{\circ} \mathrm{C}$.

After we lower the threshold, we can bring up the current to our desired. But do not put too much current into the laser, for example over $100 \mathrm{~mA}$.

If we need to build or trouble shooting the laser system, ground yourself before touch the laser. 


\subsection{Saturated Absorption System}

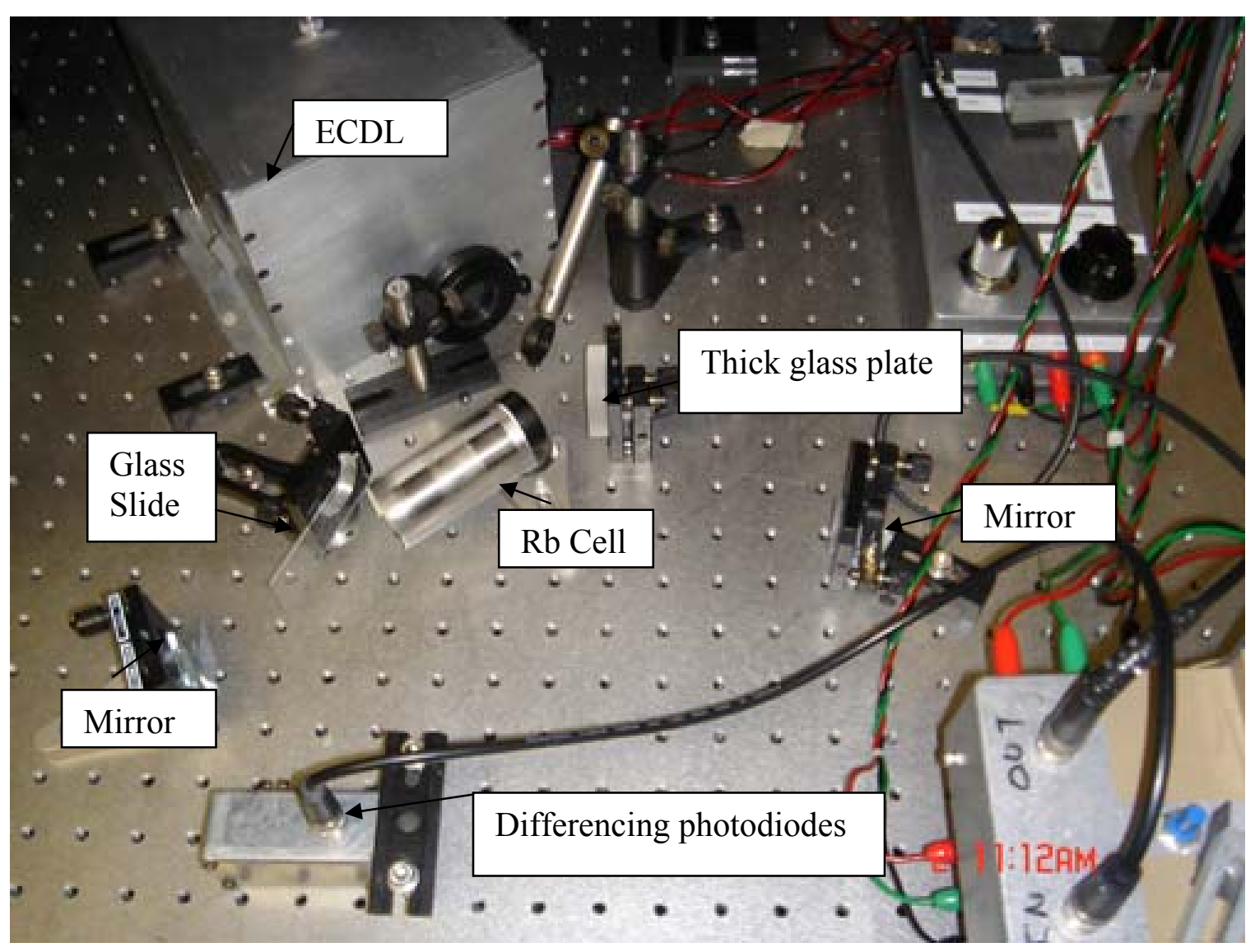

Figure 3.3: Saturated Absorption Setup

Figure 3.3 shows the set up of Saturated Absorption. The design is identical as other labs. The only change is that we put a mu metal shielding on the $\mathrm{Rb}$ vapor cell.

In the experiment, the vapor cell is shielded from earth magnetic field. We incident laser beam to the vapor cell, and tune the frequency of the laser beam by looking at the Saturated Absorption Spectrum. If the vapor cell in saturated absorption setup is affected by the earth magnetic field, the hyperfine energy levels of the $\mathrm{Rb}$ in the cell will be shifted. Thus we put a mu metal shielding on the cell so that the frequency tuning can be trust. But this is not the only reason. 
We have two sizes of coils for B ramp. The big coils are as big as z Helmholtz coils. The small coils are what we are using now (refer to 3.6). The big coils system can give us larger B amplitude than the small ones. When we run the big coils at $30 \mathrm{~Hz}$; we found a $10 \mathrm{~Hz}$ noise on the saturated absorption spectrum. The frequency of the noise changed as the frequency of the ramp changed. After we cover a mu metal shielding on the vapor cell, the noise disappeared. 


\subsection{Helmholtz Coil System}

Helmholtz coils system is to cancel earth's magnetic field. The setup of a pair of coils, which provides uniform $\mathrm{B}$ field in $\mathrm{z}$ direction, is shown in figure 3.4.1.

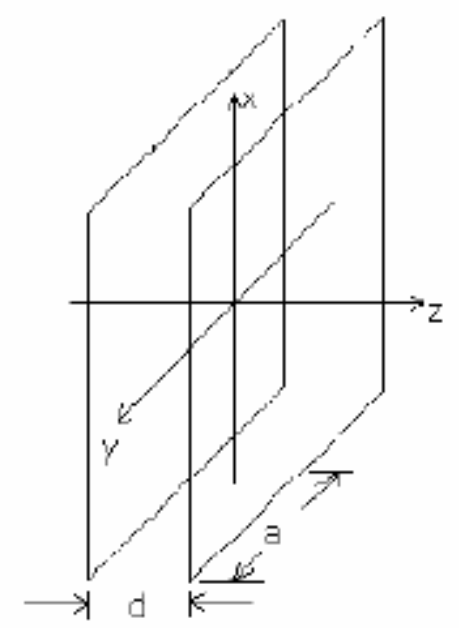

Figure 3.4.1: Setup of a pair of coils; a is the half-side of the Helmholtz coil, $d$ is the separation between two coils.

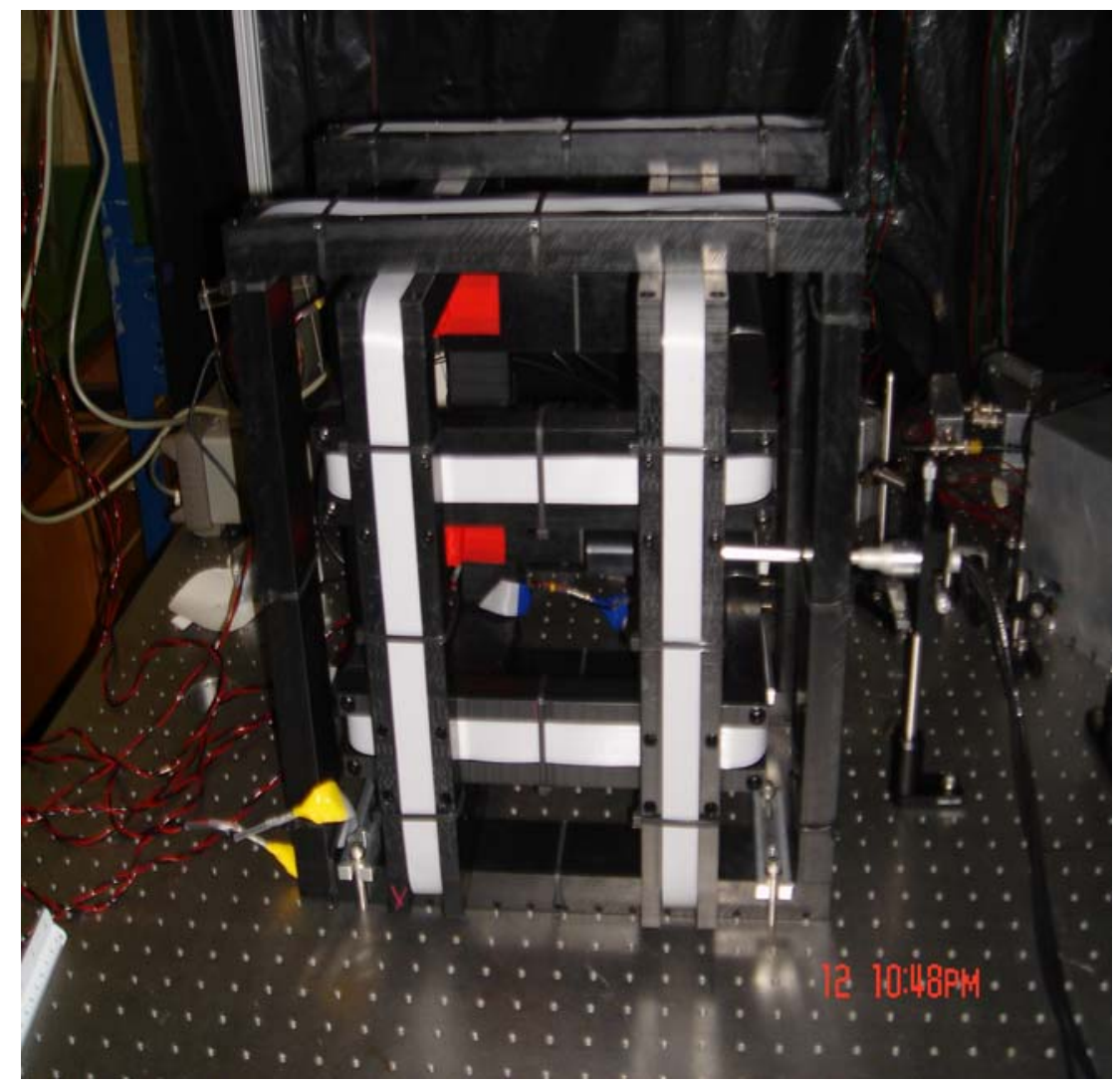

Figure 3.4.2: Setup of the three dimensional Helmholtz Coil system 
To recalibrate the Helmholtz coil system, we take off the $\mathrm{Rb}$ vapor cell from the Helmholtz coil system first. This won't destroy our previously alignment because if the laser beam is ok we can put back the cell later and just adjust the position of the cell until we see that the laser beam is in the middle on both sides of the vapor cell. Then we put a holder for the gauss meter probe and make sure that the probe in the position where we want to take the measurement. Let the coordinate system of the probe line up with the coil system's coordinate system.

Then we turn on the gauss meter and do the zeroing for it. Use a shielding to cover the head of the probe and hit the zero buttons on the $\mathrm{X}$ channel panel while holding the Enter button. If we see the reading after zeroing is still too big, do not take off the shielding and do the zeroing again until the reading is small enough for us to use, like almost zero gauss.

Now we can take off the shielding and turn on the coil system. Keep the voltage regulating knobs at their maximum and adjust the current control of each coil to get zero magnetic field. We usually make the current start from zero and then increase it very slowly. Our current regulator is not sensitive and hard to do very sensitive adjustment. Thus we need to touch it really slightly and slowly.

Sometime after several seconds the current maybe drift away a little bit from the set up current, so we need to keep an eye on it all the time. If it drifts too much then we need to do the calibration once again. Normally the calibration should do every 15 minutes. But we can reduce it depending on the data taking time. 
Here are the data showing the uniformity of $X$ coils,
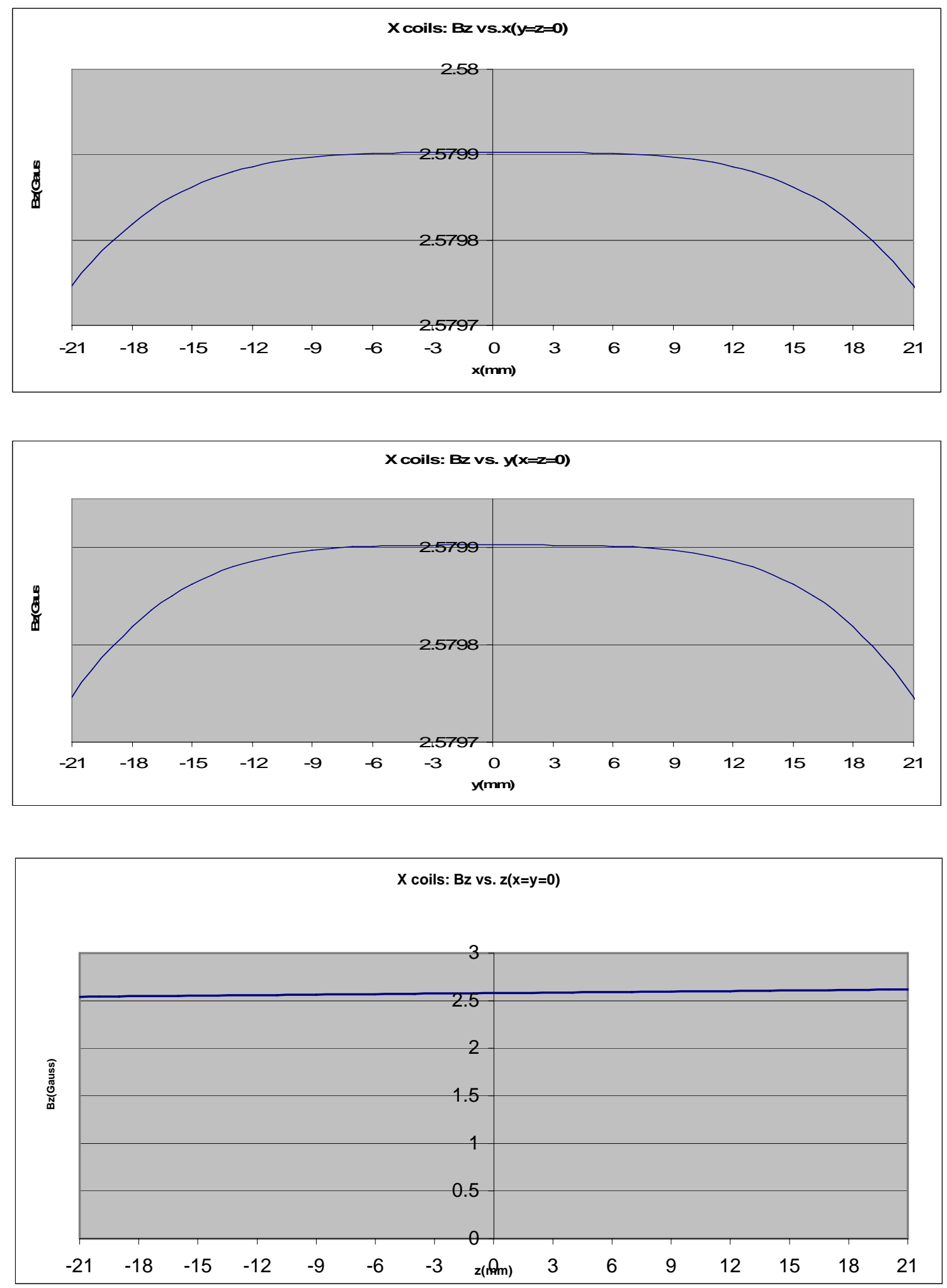


\subsection{B-ramp system}

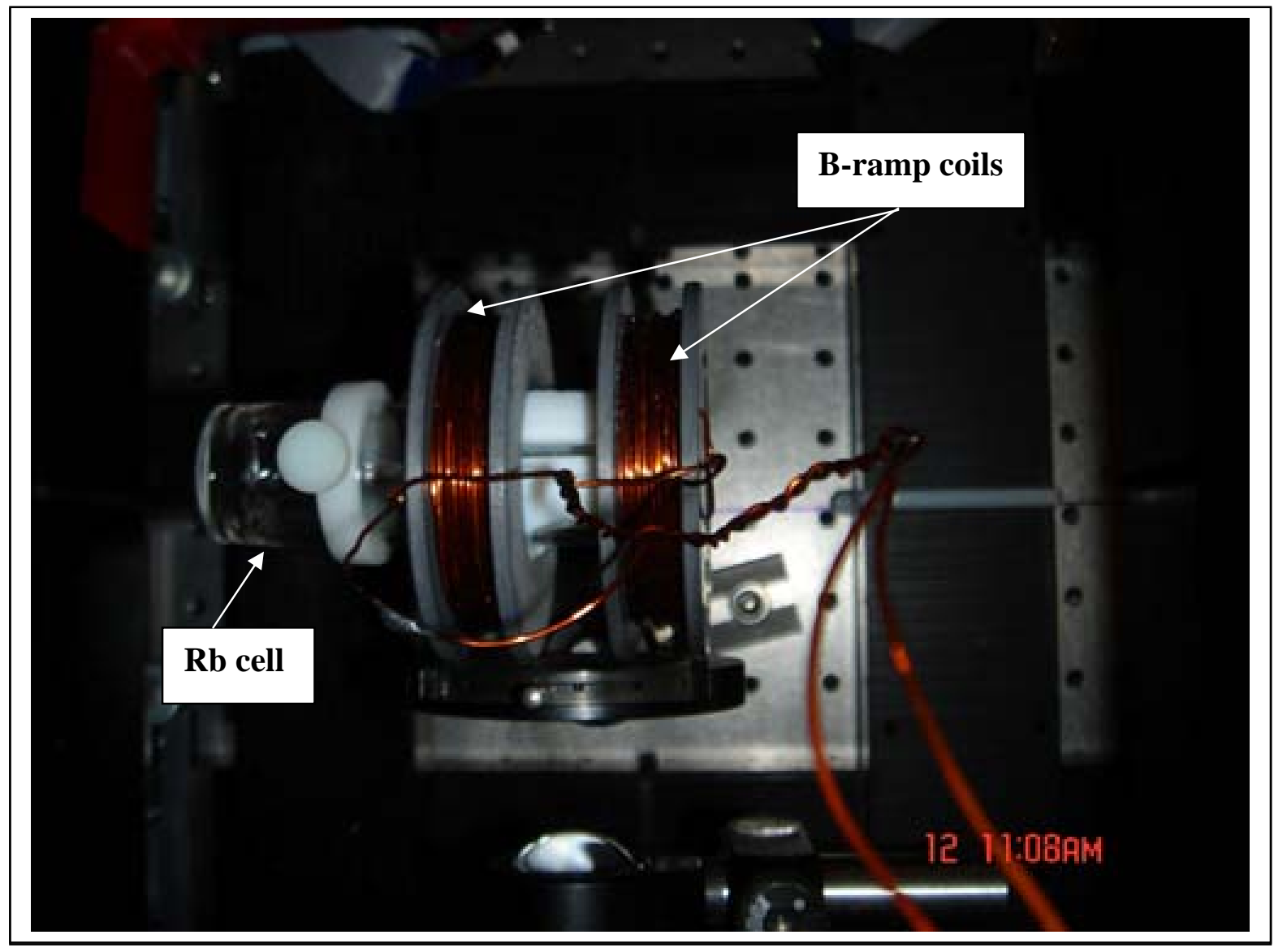

Figure 3.5: Setup of B-ramp Coils system

Figure 3.5 shows the setup of the B-ramp coils. We made a plastic holder on the cell to hold the coils. All the material we are using in the magnetic system should be nonmagnetic to prevent eddy currents. The coils are in Helmholtz configuration. We run current in same direction in the coils. Then we scan the amplitude of the current using a triangle wave. The frequency is $1 \mathrm{~Hz}$. We put 100 loops magnetic wire on each coil. Because the current source we are using is a function generator (hp. HEWLETT PACKARD 33120A), it cannot give us very high current. Thus we increase the loop. When choose magnetic wires, first check the gauge of it online, and make sure it can hold enough current that the system requires. The mean radius of the coils is $1.8 \mathrm{~cm}$. We put a $50 \Omega$ resistor in series with the coils. An oscilloscope (Tektronix TDS 714 L) is used to monitor the voltage across the coils. The maximum current through the coils is $0.058 \mathrm{~A}$. 
The amplitude of the maximum of the magnetic field is $B=\frac{\left(\frac{4}{5}\right)^{1.5} \mu_{0} n I}{R}=2.9 * 10^{-4} T=2.9$ Gauss, which means we have a B-ramp of \pm 2.9 Gauss . $\mathrm{R}$ is the radius of the coils, $\mathrm{n}$ is the number of the loops, $\mathrm{I}$ is the current, $\mu_{0}=0.000126 \mathrm{Tcm} / \mathrm{A}$ 


\subsection{Operations of experiment}

This section is about the sequence of operations for the experiment.

\subsubsection{Laser \& Saturated Absorption}

First, turn on the temperature controller, set up the control temperature for the laser. Currently we are using $26^{\circ} \mathrm{C}$. But this may change for the different seasons.

Then turn on the laser. On the current regulator, first turn on the electronics, and then turn on the laser. When turn off the current regulator, first turn off the laser, and then electronics. There are two knobs for the current tuning. One is coarse tuning, the other one the fine tuning. If we want to turn on or off the current regulator, we should start with these two knobs at the minimum. When tuning current, we change the scales slowly. Usually wait for 2 or 3 seconds in between current adjustments. Never go too high. Let the laser run for about half an hour.

Next, we should lower the threshold of the laser (refer to 3.2.3). We keep the coarse tuning of the current regulator at the minimum, and then slowly increase the fine tuning. We put a white paper card at the output of the laser and then we use a viewer to look at the output. After lowering the threshold, we increase the current to $81 \mathrm{~mA}$ to find resonance on $\mathrm{Rb}$ cell in the Saturated Absorption setup. This current also may be changed due to temperature changes.

Then we turn off the light and tune the horizontal knob for adjusting the diffraction grating in ECDL while looking at the Rb cell in the Saturate Absorption setup. Turn the horizontal knob about $1 / 4$ round clockwise from the minimum threshold setting to find resonance of the $\mathrm{Rb}$ in the cell. Do not go too far because this may change the threshold, and then we need to lower the threshold again.

After find the resonance, we tune the horizontal knob until we see the Saturated Absorption spectrum on the oscilloscope. We can also change the current back and forth to fine-tune the spectrum. The difference is that the first way can change the output 
direction the laser beam, and the second way won't. Then put the mu metal shield on (refer to section 3.3). If we find mode hops on the spectrum, and we can not use current knob to get rid of it, we need to adjust horizontal screw to do that.

The saturated spectrums will look like the following.
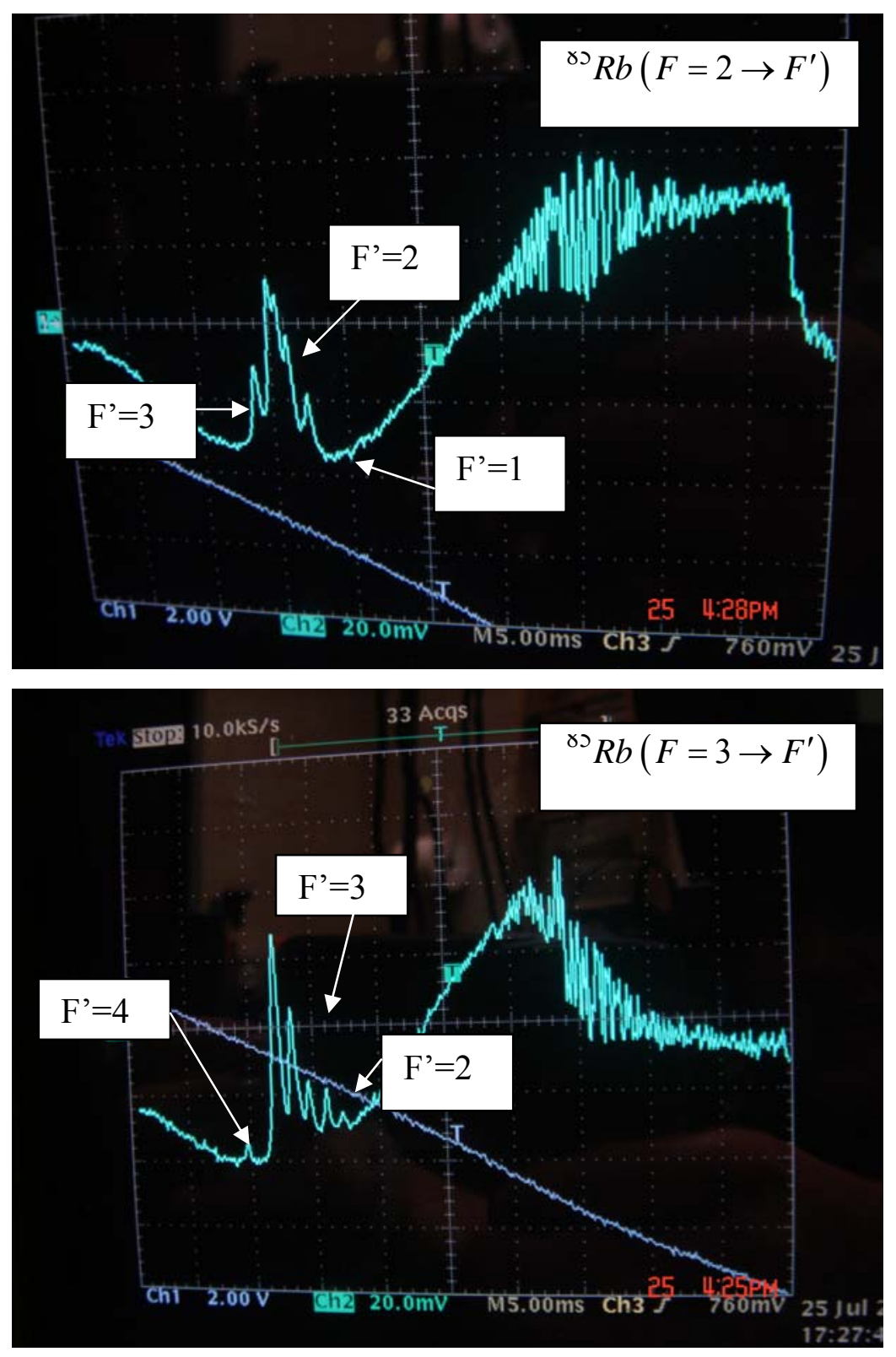

Figure 3.6.1: Saturated absorption spectrums of ${ }^{85} \mathrm{Rb}$. The non-labeled peaks are the crossover peaks. 

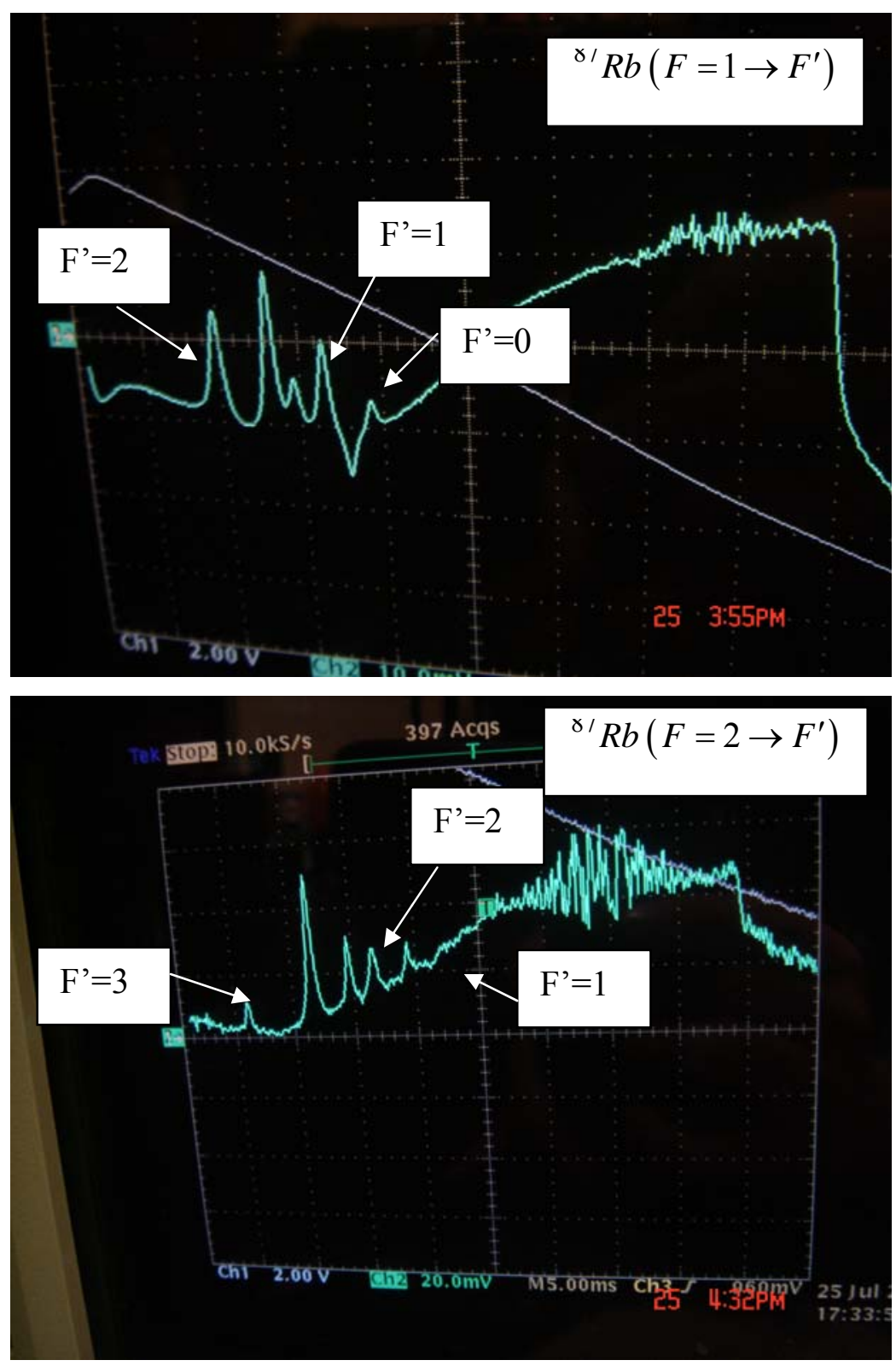

Figure 3.6.1: Saturated absorption spectrums of ${ }^{87} \mathrm{Rb}$. The non-labeled peaks are the crossover peaks. We used average function when took ${ }^{87} R b\left(F=1 \rightarrow F^{\prime}\right)$ data. The average time was 50 . 


\subsubsection{Hanle experiment}

First we need to check whether the laser beam incident directly into the vapor cell in the experiment. Make sure the vapor cell is in the right position. It should in the middle of the coil system and aligned parallel or perpendicular to the sides of the coils. Then check both sides of the vapor cell, and make sure on both sides the laser beam hits the middle. The front side is ok does not mean that the back side is fine. If the laser beam is not in the middle on at least on side of the cell, we need to change the position of the laser beam. We have two mirrors (S1 \& S2, refer to figure 3.1). If we want to move the spot up/down, first tune the vertical knob of S1 down/up, and then tune the vertical knob of S2 up/down. If we want to move the spot to the right/left, first tune the vertical knob of S1 to the left/right, and then tune then vertical knob of S2 to the right/left.

After this, we need to check the size of the incident beam. Now we are using diameter equals to $20 \mathrm{~mm}$ as the size of the incident beam. Because we cannot move the optics components in the set up, but the components are closed to each other, we cannot put a ruler in to measure the size of the beam. We made several diameter testers. They are made by paper slide. We just need to put them into the Iris near to the $\mathrm{Rb}$ cell. Be careful not the touch other components when you try to adjust something. Otherwise the laser path will no longer lined up.

After choose the size we want, we need to measure the incident intensity of the laser beam. The incident intensity is chosen by the theoretical calculations [6]. CPT will become sharper when the intensity is lower. Because of power broadening, the condition of CPT can be satisfied near $B=0$, not just at $B=0$.

We put a short focus lens and focus on to a wave meter before the Rb cell. Before we do this measurement, we need to take the background signal and do zeroing. Then we unblock the laser beam and take the reading without ramping the laser. To change the incident intensity of the beam we can adjust the half wave plate (WP2, see figure 3.1). If we want the intensity to be $0.1 \mathrm{~mW} / \mathrm{cm}^{2}$, when the size of the beam is $20 \mathrm{~mm}$, the area A 
equals $3.14 \mathrm{~cm}^{2}$. Then the power, which equals to $0.314 \mathrm{~mW}$, should equal to intensity multiply by the area A. Experimentally, we can achieve $0.01-1 \mathrm{~mW} / \mathrm{cm}^{2}$.

Next, we need to take off the wave meter probe and the lens for the probe carefully and do the recalibration for the Helmholtz coil system (refer to 3.4).

After we make the coil system working well, we can put back the vapor cell as I mentioned before. Then turn on the B-ramp (section 3.6), set the frequency equal to $1 \mathrm{~Hz}$. The amplitude equals to $10 \mathrm{~V}$. Use the triangle wave form. The reason that we like triangle wave is that triangle wave can ramp the B-field linearly.

At last we choose one transition of $\mathrm{Rb}$ and stop ramping the laser. Then use DC coupling to look at fluoresce and transmitted signal. Do not use AC coupling because this will make the signal become derivative. For a high gain of the current to voltage converter we used $10 M \Omega$ resister. 


\section{Chapter 4 \\ Data}

This chapter shows our experimental investigations of fluorescent and transmitted signal (figure 4.1\&4.2) on different transitions of Rubidium using Hanle configuration. The ripple coupled on the signal is $60 \mathrm{~Hz}$. All the data was taken at maximum intensity $0.3 \mathrm{~mW} / \mathrm{cm}^{2}$. The preliminary data we took last summer also used $0.3 \mathrm{~mW} / \mathrm{cm}^{2}$ as incident intensity.

For the EIT fluorescence signals,

on the ${ }^{85} R b F=2 \rightarrow F^{\prime}=2$ transition, the FWHM is $320 \mathrm{mG}$ in the preliminary data, and is $487 \mathrm{mG}$ in figure 4.1 ;

on the ${ }^{87} R b F=1 \rightarrow F^{\prime}=1,2$ transition, the FWHM is $202 \mathrm{mG}$ in the preliminary data, and is $278 \mathrm{mG}$ in figure 4.2 .

In reference [7], the incident intensity is $21 \mathrm{~mW} / \mathrm{cm}^{2}$, which is much higher than ours; the FWHM is around $30 \mathrm{mG}$ on ${ }^{85} \mathrm{RbF}=2 \rightarrow F^{\prime}=2$ transition, which is much lower than our results. Quote from reference [7]:"With the laser output power (up to $2 \mathrm{~mW}$ ) and experimental sensitivity (better than $0.1 \%$ ) available, no sub-natural width structure has been observed in the fluorescence arising from the $F_{g}=1$ of ${ }^{87} R b$, possibly due to the low probability of the all hyperfine transitions." But we found EIT signal on the ${ }^{87} \mathrm{RbF}=1 \rightarrow \mathrm{F}^{\prime}=1,2$ transition with good SNR.

\begin{tabular}{|c|c|c|c|c|c|}
\hline \multirow{2}{*}{} & \multirow{2}{*}{$\begin{array}{c}\text { Incident } \\
\text { Intensity }\end{array}$} & \multicolumn{2}{|c|}{${ }^{85} R b F=2 \rightarrow F^{\prime}=2$} & ${ }^{87} R b F=1 \rightarrow F^{\prime}=1,2$ \\
\cline { 3 - 6 } & & FWHM & Contrast & FWHM & Contrast \\
\hline Recent Data & $0.3\left(\mathrm{~mW} / \mathrm{cm}^{2}\right)$ & $487 \mathrm{mG}$ & & $278 \mathrm{mG}$ & \\
\hline $\begin{array}{c}\text { Preliminary } \\
\text { Data }\end{array}$ & $0.3\left(\mathrm{~mW} / \mathrm{cm}^{2}\right)$ & $320 \mathrm{mG}$ & $28.36 \%$ & $202 \mathrm{mG}$ & $37.5 \%$ \\
\hline Data of ref. [7] & $21\left(\mathrm{~mW} / \mathrm{cm}^{2}\right)$ & around $30 \mathrm{mG}$ & $1 \%$ & & \\
\hline
\end{tabular}

Table 4.1: Comparison among Recent Data, Preliminary Data, and Data of ref. [7] 

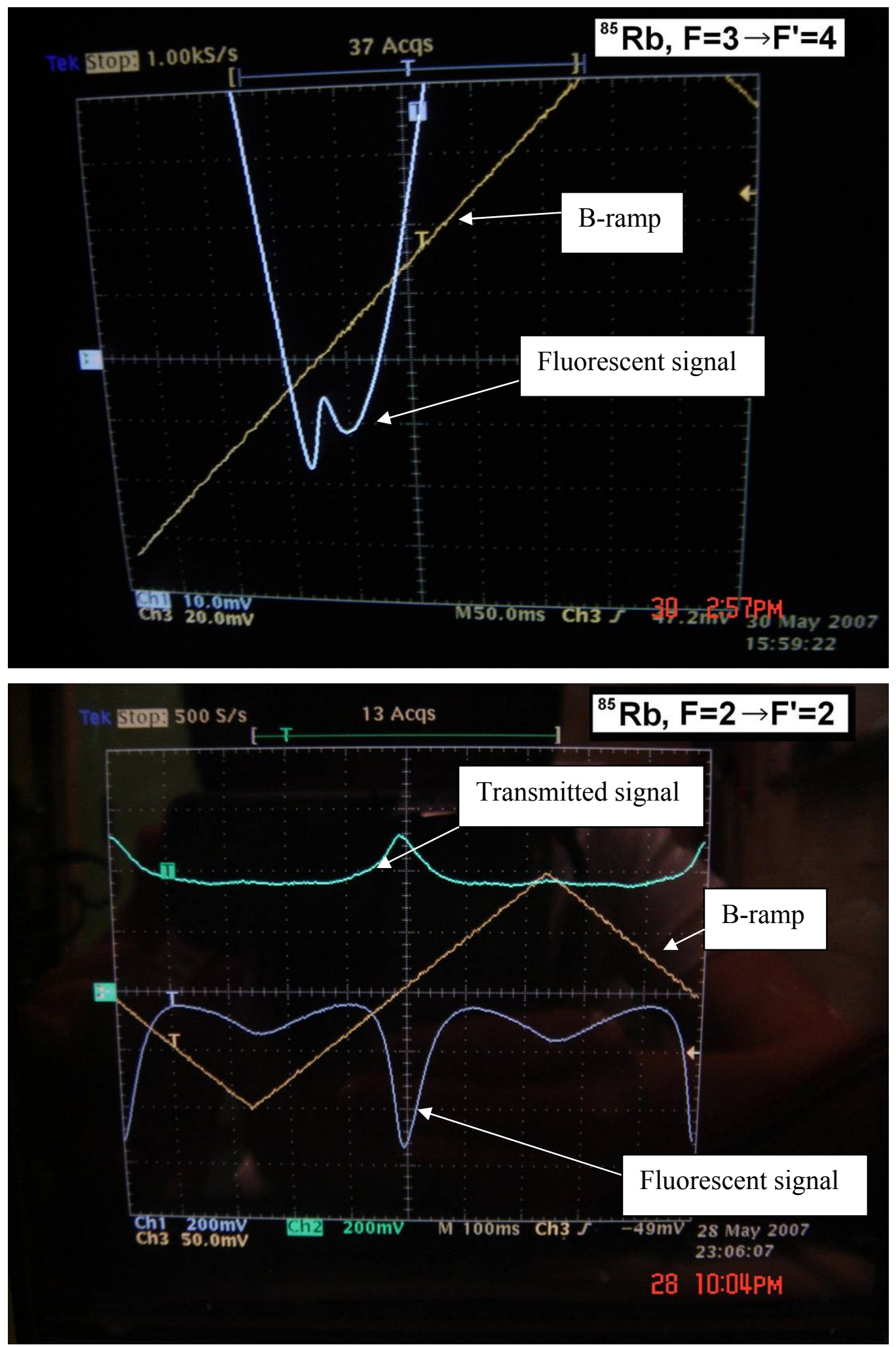

Figure 4.1: experimental investigations of fluorescent and transmitted signal on different transitions of ${ }^{85} \mathrm{Rb}$ using Hanle configuration. 

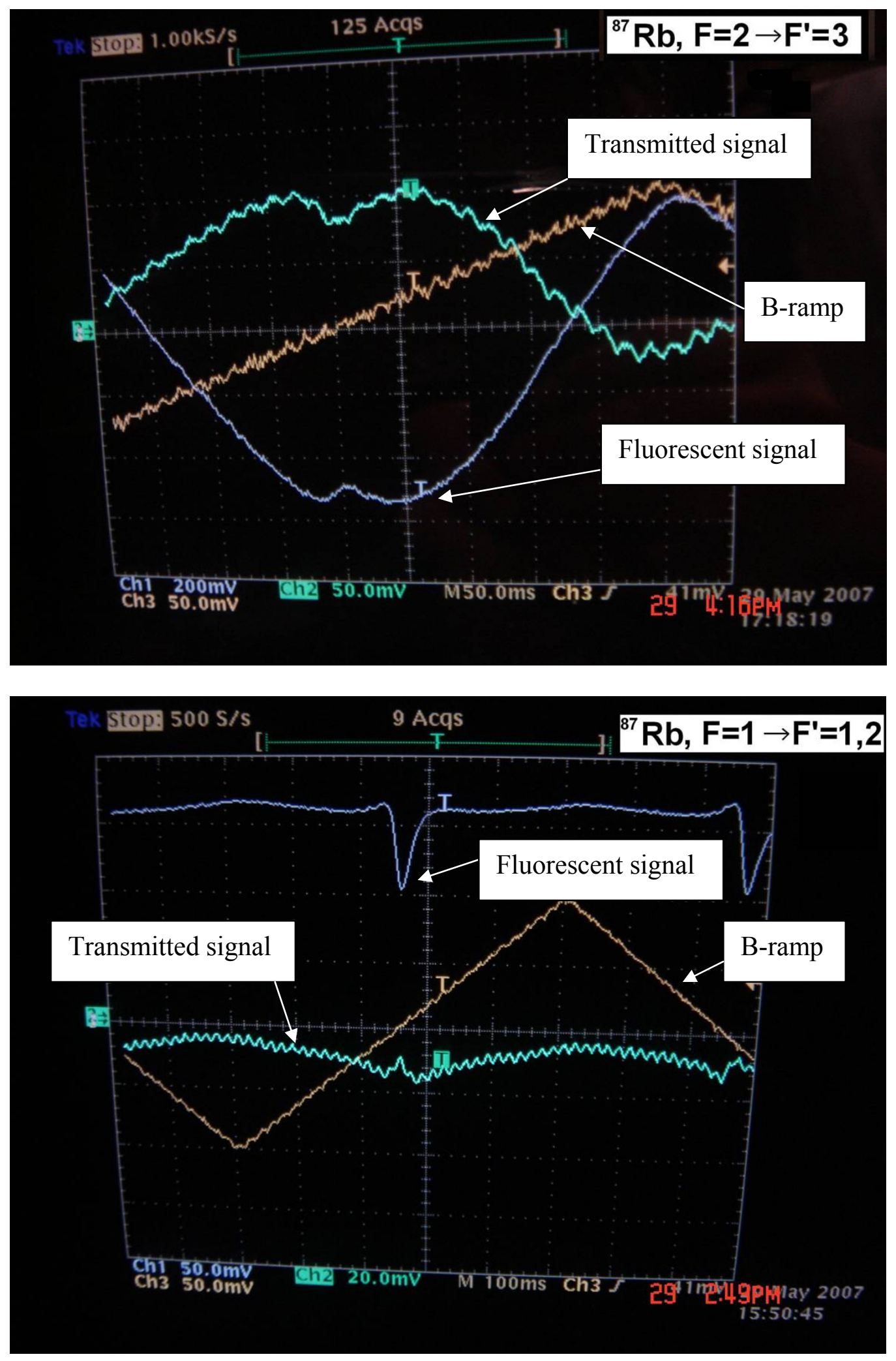

Figure 4.2: experimental investigations of fluorescent and transmitted signal on different transitions of ${ }^{87} \mathrm{Rb}$ using Hanle configuration. 


\section{Chapter 5}

\section{Conclusion \& future outlook}

\subsection{Conclusion}

In our measurements we have obtained high signal-to-noise ration (SNR) thus enabling a first measurement of EIT involving the Zeeman sub-levels of the $F_{g}=1$ hyperfine ground state of ${ }^{87} \mathrm{Rb}$. Our data on the $F_{g}=1 \rightarrow F_{e}=1$ transitions of ${ }^{87} \mathrm{Rb}$, displays the clear signature of EIT, namely a dip in the fluorescence (and hence absorption) near $\mathrm{B}=0$, accompanied by an increase in the transmission of the incident laser beam. While such a signature has been observed previously on the $F_{g}=2 \rightarrow F_{e}=2$ transition of ${ }^{85} \mathrm{Rb}$, EIT has never been observed (due to the low SNR in prior experiments [7]) on $F_{g}=1 \rightarrow F_{e}=1$ transition of ${ }^{87} \mathrm{Rb}$, which is a much weaker transition.

But we also have problems for EIA data compared to reference [15]. The reason that the Zeeman peaks in EIA are in opposite direction is unexplained.

\subsection{Future Outlook}

Our goal is to use the Hanle effect as a sensitive measure of radiation trapping [16].

To make a quantum computer is an inviting idea for the scientists nowadays. People tried lots of ways to build feasible models so that they can control information using coherently preparation systems.

Incoherent feature in light-atom interactions is a disaster potentially for available quantum computers. The incoherent effect of atoms can be studied using radiation trapping [17]. Radiation trapping is known as reabsorption of a spontaneously emitted photon. In 2001, radiation trapping effects are found in electromagnetically induced transparency experiments by Matsko, Novikova, Scully, and Welch [17]. In EIT experiments, the atomic samples are prepared coherently. Spontaneous emission is incoherent. Evidently radiation trapping gives us barrier to prepare a coherent atomic 
system. By tracking the EIT signals, we hope to build a sensitive indicator for radiation trapping, which is a very important consideration for making quantum computers.

\subsubsection{Radiation Trapping}

\section{Photon is Emitted}

\section{Atom}

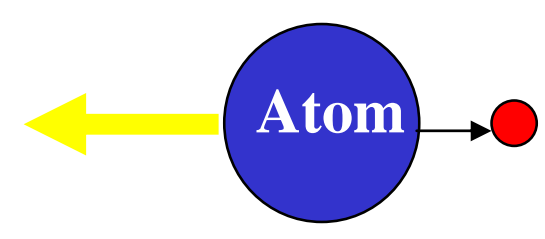

Atomic Recoil
Photon is

Absorbed

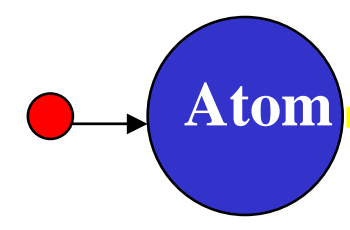

\section{Second Atom Recoils Also}

Figure 5.2.1: Radiation Trapping

Figure 5.2.1 shows a basic example of radiation trapping. In the example, we have a twoatom system. When we incident coherent laser beam onto the system, the atoms are coherently pumped from ground states to the excited states and back. However, the atoms are also spontaneously emitting photons. The emitted photons have a chance to be absorbed by a neighboring atom. If the neighboring atom absorbs the photon emitted from the other atom, the radiation energy has been trapped inside the system. This phenomenon is called radiation trapping.

The energy absorption causes the recoils of the two atoms in opposite directions. The recoil causes heating while the repulsion decreases density. Thus radiation trapping limits the density [18] and temperature of the atomic system [17]. T. Walker, D. Sesko, and C. Wieman [19] found that radiation trapping induced abrupt changes in the atomic cloud shape under certain circumstances. 
Furthermore radiation trapping causes incoherent excitation of the atoms [18]. Because the emitted photons have random phase and polarization, so called incoherent, when the atom absorbs these incoherent photons, it can be incoherently pumped to the excited states. Thus the coherence of the atomic system will be decreased.

We know that when we increase the temperature, the atomic density will increase. It is expected to cause a change in Radiation Trapping. As we explained above, Radiation Trapping leads to incoherence in the atomic system. Therefore Radiation Trapping is expected to cause change of linewidth and contrast in the Electromagnetically Induced Transparency (EIT) that we can create in an atomic sample. This is because EIT is a coherent effect- $\mathrm{a}$ consequence of coherent population trapping (CPT) in an atomic system. 


\section{References}

1. Michael Fleischhauer, Atac Imamoglu, and Jonathan P. Marangos, "Electromagnetically induced transparency: Optics in coherent media", REVIEWS OF MODERN PHYSICS, VOLUME 77, APRIL 2005

2. M. Scully, S.-Y. Zhu, A. Gavrielides, Phys. Rev. Lett. 62(1989) 24.

3. E. Arimondo, "Coherent population trapping in laser spectroscopy" in Progress in Optics 35, 257 - 347, E. Wolf, ed. (Elsevier, Amsterdam, 1996).

4. A. Lezama, S. Barreiro, and A. M. Akulshin, "Electromagnetically induced absorption", Phys. Rev. A 59, 6, JUNE 1999

5. A.M. Akulshin, S. Barreiro, and A. Lezama, Phys. Rev. A 57, 2996 (1998).

6. F. Renzoni, W. Maichen, L. Windholz, and E. Arimondo, "Coherent population trapping with losses observed on the Hanle effect of the D1 sodium line" , Phys. Rev. A 55,3710-3718 (1997).

7. Y. Dancheva, G. Alzetta, S. Cartaleva, M Taslakov, Ch. Andreeva, "Coherent effects on the Zeeman sublevels of hyperfine states in optical pumping of Rb by monomode diode laser", Opt. Commun.178, 103 - 110 (2000).

8. W. Hanle, Zeitschrift fur Physics 30, 93(1924)

9. W. Hanle, Z. Phys. 30, 93 (1924);"The Hanle Effect - Level crossing”, translated from Hanle's memoirs in Z. Phys. D 18, 3-4 (1991).

10. see, for example, D. Budker, D. F. Kimball, and D. P. DeMille, Atomic Physics (Oxford University Press, 2003), Section 3.9.

11. Albert Messiah, "Quantum Mechanics”, Vol. II, 573(1996)

12. Albert Messiah, "Quantum Mechanics”, Vol. II, 1056(1996).

13. C. Wieman and L. Hollberg, "Using diode lasers for atomic physics," Rev. Sci. Inst. 62, 1 20 (1991).

14. K.B. MacAdam, A. Steinbach, and C. Wieman, "A narrow-band tunable diode laser system with grating feedback, and a saturated absorption spectrometer for Cs and Rb", Am. J. Phys., Vol. 60, No. 12, December 1992 
15. F Renzoni, C Zimmermann, P Verkerk and E Arimondo, "Enhanced absorption Hanle effect on the $F_{\mathrm{g}}=\mathrm{F} \rightarrow \mathrm{F}+1$ closed transitions", J. Opt. B: Quantum Semiclass. Opt. 3(2001) S7-S14.

16. K. Ellinger, J. Cooper, and P. Zoller, Phys. Rev. A 49, 3909 (1994).

17. A. B. Matsko, I. Novokova, M. O. Scully, and G. R. Welch, "Radiation trapping in coherent media", Phys. Rev. Lett. 87, 133601 (2001).

18. M. Fleischhauer, Europhys. Lett. 45, 659 (1999); G. Ankerhold, M. Schiffer, D. Mutschall, T. Scholz, and W. Lange, Phys. Rev. A 48, R4031 (1993).

19. T. Walker, D. Sesko, and C. Wieman, Phys. Rev. Lett. 64, 408 (1990); D. Sesko, T. Walker, and C. Wieman, J. Opt. Soc. B. 8, 946 (1991). 